\title{
MINIMALLY INVASIVE DENTISTRY AND DENTAL CARIES MANAGEMENT: A SCOPING REVIEW
}

\author{
Nada a. Alsufayan ${ }^{1 *}$ \& David Patrick ${ }^{2}$ \\ 1. Dental Health Department, College of Applied Medical Sciences, King Saud University, Riyadh, Saudi Arabia. \\ 2. Academic Unit of Restorative Dentistry, The School of Clinical Dentistry, The University \\ of Sheffield, Sheffield, United Kingdom. \\ (Received on Date: 11 March 2021 \\ Date of Acceptance: 16 March 2021 \\ Date of Publish: 19 March 2021) \\ Email:nalsufayan@ksu.edu.sa
}

\begin{abstract}
This scoping review aimed to evaluate available literature in the field of minimally invasive dentistry (MID) and to detect its implications on dental caries management in adult groups at a global level. Scoping review methodology is described as having more breadth than depth by nature, and it follows five defined framework stages to trace gaps within the existing literature. Three key terms were used: "minimally invasive dentistry", "minimal intervention dentistry" and "preservative dentistry" at three databases: Web of Science, Scopus, and Medline Via Ovid. Resulting data cleared from duplication, and settled according to inclusions and exclusions. A charting form was created to summarise and allocate data into identified themes. 642 papers initially resulted, and only 65 papers were included. The majority originated in the USA. Six themes were concluded: the concept of MID, caries risk assessment, caries removal and cavity design, adhesives/ restoratives, dental caries detection, and dental practitioner's attitude. The concept of MID theme was dominant. However, caries risk assessment theme was the least common. Much literature advocated for repairing dental restorations instead of complete replacements. Many dental practitioners seem to be aware of the MID, but only a minority applied it into their daily dental practice.
\end{abstract}

\section{Keywords}

Minimally invasive dentistry, dental caries, preventive dentistry, restorative materials, remineralisation. 


\section{Introduction}

Dental caries has affected all age groups in both developed and developing countries. Dental caries can also be called an economic burden for the high demand for restoring, replacing, preventing lesions, and other treatments to maintain functional and aesthetic perspectives. These treatments will cost governments in providing the necessary materials, constructions, professional's payment, and scientific evidence-based practice. In highincome developed countries, around 5$10 \%$ of the public health budget is spent on oral health care which may exceed the financial resources of the middle and low-income developing countries. The same situation can also occur on a small scale for an individual patient to meet his/her oral needs (Daly, 2013). Decayed teeth were commonly managed by invasive interventions such as restoration and extraction. The gold standard approach of restorative dentistry was based on G.V. Black theory that says, "extension for prevention". It was established on removing all of the demineralised tooth structure and some of the adjacent healthy tissues. Then, rebuilding it with restorative material, to prevent further caries as it was believed. However, applying preventive measures such as fluoride and pit and fissures sealant was with minimum attention in this theory. The geometries of Black's cavity designs and the innovation of ultra-high- speed rotary cutting devices enabled dentists to prepare cavities with an even further extension. This conventional method has led to weakened tooth crowns, increased tendency of restoration's replacement and persistent dental caries (Moncada et al., 2015).

With a better understanding of the dental caries process, and the conclusion that restorations do not cure decayed teeth, minimally invasive dentistry (MID) notion has been introduced. This approach can be defined as managing dental caries with maximal preservation of healthy tooth tissues through biological means rather than conventional invasive operative dental techniques. It was established on several principles such as; early caries detection, remineralisation of demineralised lesions, and repair restorations instead of replacement. Accordingly, it can be understood that MI theory and techniques are handling dental caries prior to and after its occurrence. It attempts to prevent caries lesions from developing and to manage the cavity lesions surgically, if indicated, in a minimally invasive manner. Pit and fissure sealants, microinvasive infiltration, Atraumatic restorative treatment (ART), and Preventive resin restorations (PRR) are the common and effective $\mathrm{Ml}$ techniques in managing caries lesions at different status (Frencken et al., 2012, Molina et al., 2005).Scoping review is being expanded for use in different health research fields, and it is considered a type of literature review. scoping study is a form of knowledge synthesis that explores a broader research question/s than a systematic review, and gaps in a specified research field, while maintaining clarity, iterative, and 
comprehensiveness. Arksey \& O'Malley (2005) described its aim "to map the key concepts rapidly underpinning a research area and the main sources and types of evidence available, and can be undertaken as standalone projects in their own right, especially where an area is complex or has not been reviewed comprehensively before". It initially composed of five stages; identification of the research question, identification of the relevant data, study selection, charting the data, collating, and summarising and reporting the results. It considers different study designs and a wide range of evidence. Consequently, the searching, selecting, and synthesising of the collected data will be according to a systematic manner with flexible inclusion and exclusion criteria.

Currently, it can be noticed that in the field of dentistry, the number of review papers such as narrative reviews, systematic reviews, and meta-analyses are increasing. From the literature review and results papers, the absence of any scoping review papers related to MID's implications on dental caries management was noticed, and this was a trigger for taking this project into practice. None of the available literatures were mapping the broad topic of MID and classify the key characteristics related to this concept from the perspective of dental caries management, while identifying the different types of available evidences including grey literatures. However, some of the available literatures tend to address the same concept in different dental specialties, such as endodontics and prosthodontics, which concentrate more on minimally invasive procedures rather than the implications on dental caries management. Even though there are high numbers of evidence on MID available, there is still a large gap in the application of this concept for the purpose of dental caries management that could be aided by this scoping review in defining the motives (Showkat et al., 2020, Geštakovski, 2019, Fernández et al., 2020).

This scoping review aims to evaluate and summarise the available literature that is related to the implications of the MID on dental caries management. The objectives were to:

1- Collect published literature that is concerned about MID through online health-related

databases.

2- Establish related themes of MID implications on dental caries management.

3- Summarise and discuss the resulting data within the identified themes.

4- Trace gaps in the existing literature for future considerations.

\section{Materials and Methods}

The aim is to answer the following question 'What are the implications of MID on adult's dental caries management at the global level?'. Three online databases were used; Web of Science, Scopus, and Medline Via Ovid. "minimally invasive dentistry", "minimal 
intervention dentistry", and "preservative dentistry" were the agreed and employed key terms. They were commonly used to express the same concept (Frencken et al., 2012). The pilot search strategy was applied to the key terms by using the Scopus database to confirm their appropriateness. Inclusion and exclusion criteria were developed and updated as the search progressed for more familiarity with the topic. No restricted time interval since the MID is a current concept.

The initial inclusions were:

1. All study types related to MID concept and dental caries management.

2. Papers provided in English.

3. Papers concerned adult population and permanent dentition in good health and ability.

4. Articles with online access.

The initial exclusions were:

1. Papers with title and no abstract or text available online.

2. Any papers irrelevant to MID.

3. Papers that concern the pediatric, geriatric and pregnant population.

4. Papers related to other procedures than restorative, including aesthetic.

EndNote software was used for importing, reviewing, and duplication removing. $5^{\text {th }}$

June 2017 was settled to be the deadline for the data collection process.
Each literature was inspected by each researcher independently for its connection to the inclusion and exclusion criteria-included papers transferred to a data charting form. Variables to extract were based on their potential in answering the research question. The form was tested on fifteen papers to ensure its applicability. Following data retrieval, the researchers analysed data, reported results and made them sense by developing quantitative and qualitative summary with SPSS.

\section{Results and Discussion}

A summary of the included papers was clarified in (Fig. 1). 41 papers were literature reviews, 9 papers were in vitro, 4 survey study, 3 case reports, 2 crosssectional studies, 1 invitro and invivo papers, 1 in vivo, 1 policy statement, 1 randomized controlled trial, 1 Prospective study, and 1 systematic review. Most of the papers were written by authors from USA $(n=10)$, France $(n=9)$, UK $(n=8)$, India $(n=6)$, Germany $(n=5)$, and the rest were from Australia $(n=4)$, Brazil $(n=4), \quad$ Switzerland $(n=3)$, Japan (n=2), Kuwait (n=2), Norway $(n=2)$, Turkey $(n=2)$, Argentina $(n=1)$, Canada $(n=1)$, Chile $(n=1)$, China $(n=1)$, Croatia $(n=1), \operatorname{KSA}(n=1)$, Sweden $(n=1)$, and the Netherland ( $n=1$ ) (Fig. 1).

The majority of the included papers were under the concept of a MID theme $(n=36)$, and the rest were classified as following; caries risk assessment (CRA) $(n=2)$, caries removal and cavity design $(n=10)$, adhesives and restorative 
materials $(n=8)$, dental caries detection $(n=6)$, and dental practitioner's attitude to $\operatorname{MID}(n=3)$.

\section{The scoping review:}

The scoping review has some similarities to a systematic review as they both follow a structured process in identifying and analysing all relevant literatures that pertain to the research question. However, they tend to differ in their purposes and aims. A systematic review would be adopted to sum up the available literatures that excel all others on a specific research question. It can validate the certainty and quality of the relevant evidence on a current practice. It will also define any new practices and inspect conflicting results and variations in the practice and attempt to resolve them. Both systematic and scoping reviews can be conducted with the aim of defining areas for further research, but the latter is concentrating more on knowledge gaps (Munn et al., 2018). Besides the scoping review, some would consider that literature reviews might be comparable to a scoping study as both of them provide an overview of a topic or concept. A literature review contributes by summarizing a particular topic in a subjective manner by knowledgeable and experienced authors. It establishes the new reader with the proper familiarity and understanding of the current topic before carrying out new research. Despite the few common features between them, a scoping review follows a systematic and exhaustive searching process that is preceded by a well-structured protocol. It usually aims for transparent, reproducible, and reliable conclusions by following clear steps to reduce errors (Aromataris \& Pearson, 2014, Peters et al., 2015). A scoping review has the advantage of providing useful conclusions about the nature and characteristics of existing papers in certain fields in a reasonably short time. Moreover, the identified gaps in the area of interest can be valuable for policy makers and researchers for their future considerations. It could be a useful instrument in investigating the related research design and conduct, enabling researchers to decide which areas are lacking. However, it cannot assess the quality of the primary research results and cannot evaluate the effectiveness of certain interventions. A scoping review is accounted as a narrative approach of existing evidence, and cannot provide critically appraised answers to a particular question (Arksey \& O'Malley, 2005, Peters et al., 2015, Daudt et al., 2013).

\section{The concept of MID}

The concept of MID was a comprehensive theme that summarises the main principals, scientific bases, and practical aspects of MID. Some literature focused on different materials, biological approaches, and preventive measures in managing dental caries and considering its position and activity (Kanzow et al., 2016). However, a case report suggested that prevention is the most cost-effective measures for preserving natural teeth (Malterud, 2012). ART was an important 
part in MID; some articles found a relation between the proper case selection and use of the high viscosity Glass ionomer cement (GIC) and lower dental caries incidence (Molina et al., 2013). One review stated that ART is a reliable and efficient technique in both developing and developed countries. However, the older dentist could not accept it as related to their traditional education (Holmgren et al., 2013). Remineralisation of demineralised dental tissues is another principal in MID.

However, only one article discussed it in details. Hemagaran and Neelakantan (2014) indicated that understanding demineralisation/remineralisation process allows the dentist to treat lesions before their progression. Agents such as fluoride, Xylitol, CPP-ACP, bioactive glasses, and Ozone are a good example of enhancing remineralisation. A comparative technique to remineralisation called "resin infiltration" was discussed inadequately in some literature and used mainly in initial caries lesions. It stands for filling the porous enamel with resins to stop the demineralisation process. Experiences and evidence of this field are limited for the insufficient number of clinical trials. Restoration's repair or replacement was an area of controversy; however, the majority are calling for repairing of defective restorations for the purpose of longevity and suggesting the replacement to be a final option (Moncada et al., 2015). A prospective study applied to compare between composite and amalgam repair instead of replacement on one thousand participants and concluded that composite refurbishment was a costeffective decision. It also recommended several factors to consider when deciding the proper action for defective restorations such as preferences of patients and dentists, and availability of time and materials (Kanzow et al., 2016).

\section{Caries risk assessment}

This theme represented approaches that dental professionals can apply to prevent and

treat dental caries as early as possible. One literature review discussed several criteria that can help in determining the level of caries risks such as general oral health, caries development nature, amount of plaque accumulation, frequency of sugar intake, and fluoride exposure status 17 . One survey study examined the knowledge, opinions, and practices of two thousand general French dentists to CRA action, and found that they still need to expand their knowledge and to practice CRA in a daily routine. It also suggests that creating special systematic codes related to CRA can encourage its application (Doméjean et al., 2017).

\section{Dental caries detection}

The included papers have summarised the uses of different dental caries detection deceives and their implications on caries diagnosis and treatment within the concept of MID. Fluorescence 
technology was a commonly used $\mathrm{Ml}$ evaluating system. It was produced into different devices such as; Sporolife, DIAGNOdent, and Vista proof. One case report found that Vista proof was more specific and DIAGNOdent was more sensitive in detecting occlusal caries (Betrisey et al., 2014). Sporolife system showed some efficiency in detecting infected and affected dentine and differentiating between them (Erol et al., 2014). Fried (2010) reviewed the efficiency of optical trans-illumination, fluorescence, and optical coherence tomography devices on the early detection of dental caries. They concluded that $x$-ray is usually not sensitive enough in detecting early lesions. However, optical coherence tomography tends to show them more clearly. They also added that when selective removal of dental caries is required, a combination of different imaging methods could be useful. Guerrieri et al., (2012) reviewed different methods and devices that can help in the early detection of dental caries and proposed that CRA would be an effective measure. They argued certain criteria such as lesions extent and status, and their efficiency in planning and

predicting $\mathrm{Ml}$ treatment and traditional caries diagnostic techniques such as intra-oral examination and radiographs are still the most used and efficient methods, and could not be substituted by the new technologies due to their practicality and feasibility. They lastly added that fluorescence technologies would be useful in educating and motivating patients.

\section{Caries removal and cavity design}

Many papers introduced the function of different air abrasions, lasers, and chemical agents in the MI caries removal. They also discussed the impact of these techniques on dental hard tissues at different surfaces. For instance, literature argued that the use of sonic and ultrasonic cavity preparation systems as an alternative ultraconservative procedure seems to be useful in preventing marginal ridges failure, especially in class Il restorations (Koubi \& Tassery, 2008).

Air abrasion was acknowledged to be more promising $\mathrm{Ml}$, preparatory technology, especially when applied by knowledgeable practitioners who have sufficient clinical experience (Farooq et al., 2013). Aluminium was the conventional abraded particles for many years but tend to be intense on tooth structure, and dental practitioners should be selective when applied it clinically. More advanced bioactive glasses were introduced and showed promising selective removal of demineralised areas without affecting sound dental tissues. It also showed a cleaning of extrinsic stain that helps in detecting caries, easily and precisely. However, it considered slower than aluminium abrasion (Faroog et al., 2013).

Dental lasers presented an acceptable conservative cavity preparation, particularly for the classical geometry (box-shaped). They are cheaper, faster, and more convenient. However, they may disturb the adhesion process, 
when specially designed cavities required as they will result in more rectangular and triangle cavities (Savatier et al., 2014).

Chemo-mechanical caries removal
agents advised being used alternatively to conventional rotatory instruments for conservative purposes. Carisolv and Papacarie are a good example, but the latter provided shorter excavation time and better morphological features of remaining dentine that enhanced bonding action (Hamama et al., 2013).

\section{Adhesives and restorative materials}

This theme identified the efficiencies, properties, and clinical considerations of some

adhesive systems, resins, and cement within the concept of MID. Current sealants were developed in colors to assess their presence easily and inconsistencies to enhance their retention, that can result in providing higher caries prevention. PRR showed promising outcomes when applied with resin-based sealant than GIC due to its superior retention (Frencken et al., 2012).

An updated form of GIC called apatite ionomer cement (AIC) has enhanced the remineralisation activity. An invitro has investigated the mechanical strength and ion release of different GIC samples mixed with porous and spherical hydroxyapatite under different conditions. AIC resulted in higher fluoride release and better compressive strength that promote caries prevention, including secondary caries (Chiu et al., 2017). Adhesives have helped in embracing the concept of MID by providing adhesion to tooth coloured restorations in the conservatively prepared dental cavity. It is preferable for adhesives to be applicable to the newer generations of bonding agents. One invitro found that they may show some microleakage, especially at the gingival margin with class $V$ restoration. It also noticed that using of self-etch system appeared to cause least micro-leakage even at the gingival margins when comparing it to other systems (Gupta et al., 2017). In regard to the restorative materials, resin composites would be the proper material with the right compositions and properties. It is strongly suggested that dental practitioners should follow both manufactural and evidence-based guidelines when applying restorations for safety and efficiency purposes (Lynch et al., 2014).

\section{Dental practitioner's attitude to MID}

General dentist's position on the concept and adoption of MID for dental caries management has been expressed in this theme. Two crosssectional studies applied to general dentists from different countries, continents, and beliefs. It was found that their awareness and practices of $\mathrm{Ml}$ dental approaches into their daily routine were deficient. They believed that MID is a temporary, inferior practice and the conventionally invasive procedures considered the 
actual dental treatment (Shah et al., 2016).

In a survey study, young dentists showed higher acceptance of $\mathrm{Ml}$ treatments. The

majority of participants revealed that they started restorative treatment when lesions became advanced. In addition, they showed some needs for more training courses on the proper $\mathrm{MI}$ interventions (Rechmann et al., 2016).

\section{Conclusion}

This scoping review has reviewed the available literature that is related to MID implications on dental caries management by using three different databases with three different key terms. Consequently, six related themes have been formulated to help in answering the research question. Within the limitations of this scoping review, some of the important related literature has been loosened since it needs more specific term such as "minimally invasive dentistry AND remineralisation", which contradicted its nature "broad search terms" (Arksey \&O'Malley, 2005). Some of the literature was not found online, and this was predicted by Arksey and O'Malley (2005). Accordingly, data collection was limited to June 2017 for accessibility reasons.It is recommended to extend this scoping review to cover the latest literature in MID and dental caries management. One of the gaps that concluded form of this review is the need for more dental caries prevalence evaluation in patients who have undergone $\mathrm{Ml}$ approaches.In addition, it will be useful to enhance the radiographical examinations of mineral changes in very early lesions. Moreover, it is still required to solve the shrinkage and micro-leakage issues of the adhesive restorative materials. Further educational policies are demanded employing the concept of MID into dentistry school courses.

\section{List of figures}






Fig. 1 PRISMA flow diagram of search and screening results

\section{Reference}

Arksey, H., O'Malley, L., 2005.Scoping studies: towards methodological framework. IJSRM. 8(1):19-32.

Aromataris, E., Pearson, A., 2014. The systematic review: an overview. AJN. $114(3): 53-8$.

Betrisey, E., Rizcalla, N., Krejci, I., Ardu, S., 2014. Caries diagnosis using light fluorescence devices: VistaProof and DIAGNOdent. Odontology. 102(2):330335.
Chiv, S.Y., Shinonaga, Y., Abe, Y., Harada, K., Arita, K., 2017. Influence of Porous

Spherical-Shaped Hydroxyapatite on Mechanical Strength and Bioactive Function of Conventional Glass Ionomer Cement. Materials. 10(1):27.

Daly, BN., 2013. Essential dental public health. Second edition. ed. Oxford: Oxford University Press.

Daudt, H.M., Mossel, C.V., Scott, S.J., 2013. Enhancing the scoping study methodology: a large, inter-professional 
team's experience with Arksey and O'Malley's framework. BMC Med Res Methodol. 13(1):48.

Doméjean, S., Léger, S., Simon, A., Boucharel, N., Holmgren, C., 2017. Knowledge, opinions and practices of French general practitioners in the assessment of caries risk: results of a national survey. J Clin oral inves. $21(2): 653-663$.

Erol, S., Kamak, H., Erten, H., 2014. Evaluation of caries dentin using lightinduced fluorescence: a case report. JCDR. 8(1):297-298.

Farooq, I., Tylkowski, M., Müller, S., Janicki, T., Braver, D.S., Hill, R.G., 2013. Influence of sodium content on the properties of bioactive glasses for use in air abrasion. Biomed Mater.8(6):065008.

Fernández, C., González-Cabezas, C., Fontana, M., 2020. Minimum intervention dentistry inthe US: an update from a cariology perspective. Br Dent J. 229:483486.

Fontana, M., Gonzalez-Cabezas, C., 2012. Minimal intervention dentistry: part 2. Caries risk assessment in adults. Br Dent J. 213(9):447-451.

Frencken, J.E., Peters, M.C., Manton, D.J., Leal, S.C., Gordan, V.V., Eden, E., 2012.

Minimal intervention dentistry for managing dental caries - a review: report of a FDI task group. IDJ. 62(5):223243.
Fried, D., 2010. Lasers and optics for measuring: Tooth decay. OPN. 21(4):1419.

Geštakovski, D., 2019. The injectable composite resin technique: minimally invasive reconstruction of aesthetics and function. Jqi. 50:712-719.

Guerrieri, A., Gaucher, C., Bonte, E., Lasfargues, J.J., 2012. Minimal intervention dentistry: part 4. Detection and diagnosis of initial caries lesions. $\mathrm{Br}$ Dent J. 213(11):551-557.

Gupta, A., Tavane, P., Gupta, P.K., Tejolatha, B., Lakhani, A.A., Tiwari, R., Kashyap, S., Garg, G., 2017. Evaluation of Microleakage with Total Etch, SelfEtch and Universal Adhesive Systems in Class V Restorations: An In vitro Study. JCDR. 11 (4): ZC53-ZC56.

Hamama, H.H., Yiu, C.K., Burrow, M.F., King, N.M., 2013. Chemical, morphological and microhardness changes of dentine after chemomechanical caries removal. Austr Dent J. 58(3):283-292.

Hemagaran, G., Neelakantan, P., 2014. Remineralization of the tooth structure The future of dentistry. IJPTR. 6(2):487-493. Holmgren, C.J., Roux, D., Doméjean, S., 2013. Minimal intervention dentistry: Part 5. Atraumatic restorative treatment (ART)-a minimum intervention and minimally invasive approach for the management of dental caries. Br Dent J. $214(1): 11-18$. 
Kanzow, P., Wiegand, A., Schwendicke, F., 2016. Cost-effectiveness of repairing versus replacing composite or amalgam restorations. J of Dentistry. 54:41-47.

Koubi, S., Tassery, H., 2008. Minimally invasive dentistry using sonic and ultrasonic devices in ultraconservative Class 2 restorations. JCDP. 9(2):155-165.

Lynch, C. D., Opdam, N. J., Hickel, R., Brunton, P. A., Gurgan, S., Kakaboura, A., Shearer, A. C., Vanherle, G., Wilson, N. $\mathrm{H}$., \& Academy of Operative Dentistry European Section, 2014. Guidance on posterior resin composites: Academy of Operative Dentistry -European Section. Journal of dentistry. 42(4), 377-383.

Malterud, M.I., 2012. Minimally invasive dentistry-A biomimetic approach. JGD. 60(3):186- 187.

Molina, G.F., Faulks, D., Frencken, J.E., 2013. Suitability of ART approach for managing caries lesions in people with disability-Experts' opinion. Acta Odonto Scand. $71(6): 1430-1435$.

Moncada, G., Vildósola, P., Fernández, E., Estay, J., de Oliveira Júnior, O.B., de Andrade, M.F., 2015. Longitudinal results of a 10-year clinical trial of repair of amalgam restorations. Oper Dent. 40(1):34-43.

Munn, Z., Peters, M.D.J., Stern, C., Tufanary, C., McArthur, A., Aromataris, E., 2018. Systematic review or scoping review? Guidance for authors when choosing between a systematic or scoping review approach. BMC Med Res Methodol. 18:143.

Peters, M.D., Godfrey, C.M., Khalil, H., Mclnerney, P., Parker, D., Soares, C.B., 2015.

Guidance for conducting systematic scoping reviews. Int J Evid Based Healthc. $13(3): 141-6$.

Rechmann, P., Doméjean, S., Rechmann, B.M., Kinsel, R., Featherstone, J.D., 2016.

Approximal and occlusal carious lesions: Restorative treatment decisions by California dentists. JADA. 147(5):328338.

Savatier, L., Curnier, F., Krejci, I., 2014. Micro-CT evaluation of cavities prepared withdifferent Er: YAG handpieces. EJPD. 15(1):95-100.

Shah, A. H., Sheddi, F. M., Alharqan, M. S., Khawja, S. G., Vohra, F., Akram, Z., Faden, A. A., \& Khalil, H. S, 2016. Knowledge and Attitude among General Dental Practitioners towards Minimally Invasive Dentistry in Riyadh and AIKharj. JCDR. 10(7), ZC90-ZC94. 
Showkat, N., Singh, G., Singla, K., Sareen, K., Chowdhury, C., Jindal, L., 2020. Minimal Invasive Dentistry: Literature Review. jcmro. 3(09):631-636. 